\title{
HPV Prevalence and Detection of Rare HPV Genotypes in Hong Kong Women from Southern China with Cytological Abnormalities
}

\author{
Ngai Na Chloe Co, Lai-On Chu, Joseph K. F. Chow, Joseph W. O. Tam, and Enders K. O. Ng \\ Molecular Diagnostics Division, DiagCor Bioscience Incorporation Ltd, Hong Kong SAR, Hong Kong \\ Correspondence should be addressed to Enders K. O. Ng; ngko@hku.hk
}

Received 30 October 2013; Accepted 19 November 2013

Academic Editors: M. Ciotti and S.-L. You

Copyright (C) 2013 Ngai Na Chloe Co et al. This is an open access article distributed under the Creative Commons Attribution License, which permits unrestricted use, distribution, and reproduction in any medium, provided the original work is properly cited.

\begin{abstract}
Human papillomavirus (HPV) has been identified as the primary cause of cervical squamous intraepithelial lesion and invasive cervical cancer. The emergence of various commercial HPV genotyping kits with different characteristics facilitates the detection of most high-risk and low-risk HPV genotypes, but the rare HPV types are usually underdiagnosed. In the present study, HPV detection was performed using the GenoFlow HPV Array Test kit (DiagCor Bioscience), which can identify 33 HPV subtypes by specific probes. Besides, a HPV consensus probe (universal probe) was designed to capture not only the 33 genotypes but also rare subtypes. Of the 1643 Southern Chinese women tested between 2012 and 2013, the HPV prevalence was 42.3\%, with HPV 52 (139/1643, 8.5\%), HPV 81 (89/1643, 5.4\%), and HPV 16 (63/1643, 3.8\%) being the most frequent subtypes detected. Among all 695 HPV-positive cases, 56 (8.1\%) cases were only detected by the universal probe, in which 5 were either ASCUS or LSIL cases. Sequencing results confirmed HPV types 30,91, and 74, and the intratypic variants of HPV 72 and 82 were present in the 5 cases. The result suggests that some rare HPV subtypes might be involved in cervical lesions.
\end{abstract}

\section{Introduction}

Human papillomavirus (HPV) infection is the most common sexually transmitted infection worldwide. To date, $170 \mathrm{HPV}$ types have been completely sequenced [1]. Phylogenetic analysis based on the L1 ORF sequences identified five evolutionary groups with different epithelial tropisms and disease associations: Alpha-, Beta-, Gamma-, Nu-, and $\mathrm{Mu}-$ papillomavirus (PV). The Alpha PVs include the low-risk cutaneous types that typically cause skin warts, the lowrisk mucosal types that are associated with benign lesions and genital warts, and the high-risk mucosal types that can cause cervical cancer [2]. Persistent infection with high-risk Alpha PVs is a prerequisite for the development of cervical cancer and its histological precursor, cervical intraepithelial lesions. Twelve HPVs $(16,18,31,33,35,39,45,51,52,56$, 58 , and 59) are classified by the International Agency for Research on Cancer as being carcinogenic to humans, with HPV 68 being recognized as probably carcinogenic. Several other HPV types $(26,30,34,53,66,67,69,70,73,82,85$, and 97) are possibly carcinogenic based on evolutionary similarity to the known cancer-causing types [3]. HPV 16 and HPV 18 are generally recognized as the most important oncogenic viruses, which account for $>70 \%$ of all cervical cancer diagnosed worldwide [4]. However, several studies have shown the involvement of rare HPV types in cervical malignancy. HPV 61 and HPV 62 were detected in $2.3 \%$ of LSILs and 3.5\% of HSILs [5]. A retrospective cross-sectional study also identified rare HPV types (include 42, 61, 74, and 91) in $1 \%$ of 8977 cases of invasive cervical cancer [6]. A recent study using PCR with MY09/11 degenerate primers followed by direct sequencing that showed $9.47 \%$ prevalence of rare HPV types in cervical lesions. In particular, HPV 54 and HPV 81 were detected in samples with histopathological findings of CIN I, HPV 61 in samples with CIN II, and HPV 81 in samples with CIN III [7]. Emerging studies suggested the potential roles of some rare HPV types in cervical lesions and cervical cancer. 
Currently, HPV genotyping is important in four main clinical applications: primary cervical screening, HPV triage of low-grade cytological abnormalities, test of cure following treatment, and resolution of uncertainties [8,9]. Molecular methods for HPV DNA detection are broadly divided into signal and target amplification assays. The Hybrid Capture 2 (HC2) system (Digene Corporation, Gaithersburg, MD, USA) and Cervista HPV HR test (Hologic, WI, USA), the FDA-approved HPV DNA tests, which detect 13 and 14 high-risk HPV types, respectively, by signal amplification methods. The Linear Array HPV Genotyping (Roche Molecular Diagnostics, Branchburg, NJ, USA) and the InnoLiPA HPV Genotyping (Innogenetics, Belgium) are based on the amplification of HPV L1 gene followed by reverse line-blot hybridization to detect 37 and $28 \mathrm{HPV}$ types, respectively. All these assays are designed to detect only the most frequent and defined high-risk and low-risk HPV subtypes. Other subtypes beyond their defined scopes are unlikely to be detectable. In order to detect more rare HPV subtypes, GeneFlow HPV array Test kit utilized a set of degenerated PGMY primers to amplify the L1 gene of HPV followed by proprietary flow-through hybridization and captured by using a universal probe with consensus sequence of HPV. The universal probe was designed to detect not only the defined panel of 33 high-risk and low-risk HPV subtypes but also outpanel rare subtypes and the variant forms of specific HPV types. In this report, we used the GenoFlow HPV assays to evaluate HPV prevalence and genotype distribution from Hong Kong women, Southern China. We also investigated whether the out-panel HPV subtypes identified were associated with their cytology results.

\section{Materials and Methods}

2.1. Study Population. Liquid-based cytology specimens from a total of 1648 Southern Chinese women were tested when they attended private sector healthcare clinics from October 2012 to September 2013, with patient consent. All specimens were screened with conventional cytology and HPV testing was undertaken using GenoFlow HPV Array Test kit (DiagCor Bioscience, Hong Kong, SAR). Five cases were excluded from the study due to the missing cytology or age information.

2.2. HPV Genotyping. GenoFlow Human Papillomavirus Array Test Kit was used for HPV genotyping. This kit has been designed for the identification of the 33 most common genotypes of HPV. The assay covers 18 currently known highrisk HPV genotypes $(16,18,31,33,35,39,45,51,52,53,56$, $58,59,66,68,73$, and 82 ) and 15 low-risk HPV genotypes (6, $11,26,40,42,43,44,54,55,57,61,70,71,72,81$, and 84 ). A universal probe $(\mathrm{U})$ is also included to detect rare HPV subtypes that are outside the 33-genotype panel. Cervical samples preserved in liquid-based cytology medium (ThinPrep; Cytyc Corp., Marlborough, MA, USA, or Surepath; BD Diagnostics-Tripath BD Biosciences, Oxford, UK) were washed once with phosphate buffered saline and subjected to DNA extraction by using the QIAamp DNA Blood
TAble 1: Patient characteristics.

\begin{tabular}{lcc}
\hline Characteristic & \multicolumn{2}{c}{ Hong Kong Chinese women $(n=1643)$} \\
& No. of patients & $\%$ \\
\hline Age (years) & & - \\
Mean & 36.9 & - \\
Median & 29 & - \\
(range) & $(15-83)$ & \\
Cytological results & & 91.6 \\
Normal & 1505 & 5.5 \\
ASCUS & 91 & 2.5 \\
LSIL & 41 & 0.4 \\
HSIL & 6 &
\end{tabular}

ASCUS: atypical squamous cells of undetermined significance; LSIL: lowgrade squamous intraepithelial lesions; HSIL: high-grade squamous intraepithelial lesions.

Mini Kit (Qiagen) in accordance with the manufacturer's instructions. The extracted DNA was mixed with the biotinlabeled primer mix and DNA Taq polymerase provided with the GenoFlow test kit; followed by the PCR amplification step using the stated thermocycling condition. The amplified products were then genotyped using the GenoFlow HPV array system. Briefly, the amplicons were denatured and hybridized to specific HPV DNA capturing probes via Flowthrough hybridization. The hybridized DNA was subjected to a stringent wash and detected by colorimetric development. A result is valid only when there are signals at the hybridization control (HC) and the amplification control (AC) [10].

2.3. Confirmation of Out-Panel HPV Genotype. For samples with positive universal probe signal but negative for HPV specific types (out-panel HPV samples), the sample extracted DNA was amplified with MY09/11 and GP5+/6+ primer systems using standard reference protocol [11, 12]. The target amplicons were purified and sequenced using corresponding primer sets. The sequencing results were then searched through BLAST program to confirm the HPV genotype.

\section{Results and Discussions}

Among the 1643 eligible Chinese women, the age was ranged from 15 to 83 years and the median age was 29 years. Patient characteristics were shown in Table 1. Based on the cytological results, 1505 (91.6\%, 1505/1643) samples were normal with no intraepithelial lesion or malignancy, $91(5.5 \%)$ were atypical squamous cells of undetermined significance (ASCUS), 41 (2.5\%) were low-grade squamous intraepithelial lesions (LSIL), and $6(0.36 \%)$ were high-grade squamous intraepithelial lesions (HSIL). Using the GenoFlow HPV Array Test, genotyping test revealed that 695 cases were positive for HPV DNA, and the overall prevalence of HPV was $42.3 \%(695 / 1643)$. The three most prevalent types were HPV 52 (139/1643, 8.5\%), HPV 81 (89/1643, 5.4\%), and HPV $16(63 / 1643,3.8 \%)$ regardless of their cervical histological results (Figure 1). Our results were in-line with 


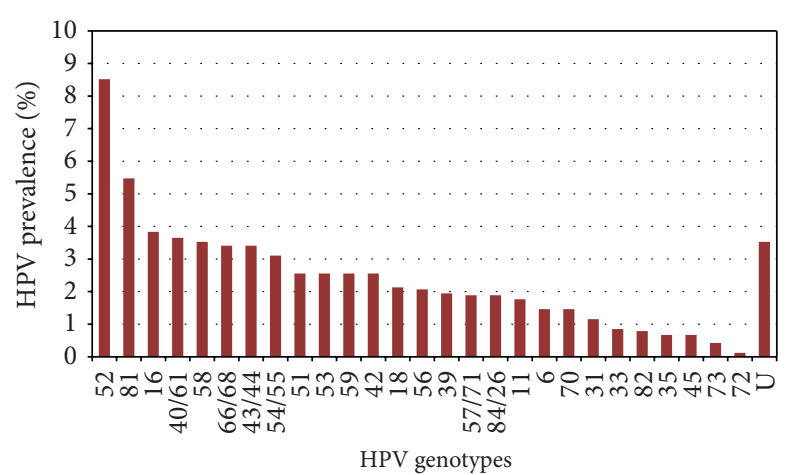

FIgURE 1: Distribution of genotypes in 1643 Hong Kong women. HPV 40 and 61, HPV 66 and 68, HPV 43 and 44, HPV 54 and 55, HPV 57 and 71, and HPV 84 and 26 share probe spots. U, cases showed positive for HPV DNA at the "universal probe" detection only, but not in-panel HPV specific types.

the reported meta-analysis studies showing that the most commonly detected HPV type in South China was HPV 52 [13-15]. Liu et al. reported that HPV 52 was the most frequently found genotype in the Guangzhou region of China while its prevalence was comparable to that of HPV 16 in Hong Kong [16]. Li et al. and Ye et al. also showed HPV 52 had the highest prevalence in Guangdong and Zhejiang of China, respectively [14, 17]. In this study, HPV 16 ranked the third and the prevalence (3.8\%) was comparable with that worldwide. The population-based type-specific prevalence of HPV 16 is ranging from around $2 \%$ to $4 \%$ in all regions [6]; however, HPV 16 infection is strongly associated with risk of cervical neoplasia and causes over 50\% of cases of cervical cancer [4]. HPV 81 (equivalent to CP8304) is also reported to be associated with precancerous or cancerous lesions $[18,19]$. The prevalence of HPV 81 in worldwide population studies is not very high but HPV 81 is one of the most frequently observed HPV types in the HIV-positive patients [20, 21]. The finding that HPV 81 prevalence was more frequent than HPV 16 in our study may be due to the geographical and biological variations in the distribution of HPV types and the sensitivities of different genotyping methods on specific HPV types.

The GenoFlow test utilized a novel universal probe to detect HPV genotypes that were outside the 33 genotype panel, which can broaden the genotype detection coverage. In the present study, 56 cases were positive for HPV DNA at the "universal probe" detection only but not in-panel HPV specific types. It suggested that one or more out panel HPV genotypes were present in the samples and the prevalence was $3.4 \%$ (56/1643), which was only slightly lower than that of HPV 16 (Figure 1). Of the 56 cases positive for unknown HPV types, $91.1 \%$ (51/56) samples presented normal cytological results in the Pap test, 3.6\% (2/56) samples were diagnosed as ASCUS, and 5.4\% (3/56) samples were LSIL. These five cases with cell abnormality were sequenced to confirm the HPV types. The genotypes were successfully identified and listed in Table 2. Rare HPV types 30,91 , and 74 as well as the variant forms of HPV 72 and 82 were detected in women with ASCUS or LSIL.
TABLE 2: Genotype distribution of HPV in the 5 cases with cell abnormalities.

\begin{tabular}{lcc}
\hline Sample no. & Cell abnormality & HPV genotype \\
\hline 16 & ASCUS & HPV 72 variant \\
21 & LSIL & HPV 30 \\
23 & LSIL & HPV 91 \\
36 & ASCUS & HPV 74 \\
44 & LSIL & HPV 82 variant \\
\hline
\end{tabular}

HPV 30, part of the alpha 6 species group along with HPV types 53,56 , and 66 , is classified as possibly-carcinogenic because of its close phylogenetic relationship with HPV 56 which is a well-known cancer causing HPV type [3]. HPV 30 is not commonly detected in the genital tract, so it is usually excluded from the commercial HPV genotyping kits. However, by DNA microarray method, Rahman et al. showed that the prevalence of HPV 30 in 342 HIV-negative Japanese women was $3.8 \%$ and was detected in 1 case of ASCUA, 7 cases of LSIL and 1 case of HSIL [22].

HPV 91 and HPV 74 belong to alpha 8 and alpha 10 species, respectively, and are both classified as noncarcinogenic [3]. Only limited studies were carried out to examine their prevalence. HPV 74 was first identified from persisting vaginal lesions of low-grade intraepithelial neoplasia of an immunosuppressed woman [23]. Choi et al. performed DNA sequencing on $209 \mathrm{HPV}$-other samples (positive on HPVPCR but negative when using specific HPV hybridization probes) to determine the HPV type. A case of HPV 74 infection was identified in LSIL patient [19]. In a cohort of 310 sexually active women in Trinidad, HPV 91 was found in 1 case by DNA sequencing but its role in cancer development is still not clear [24].

HPV intratypic variants are extensively reported, particularly in HPV 16. The oncogenic E6 and E7 proteins are commonly mutated, whereas synonymous and nonsynonymous mutations are also reported in the L1 gene in HPV 16 [2527]. Several studies investigated the genetic variability of L1, E6, and E7 genes in HPV 18, 38, 52, and 53 and showed that the intratypic variants were frequently found [28-31]. Some of these mutations increased the oncogenic potential and the infection efficiency of the viruses. Unsurprisingly, if the mutations are situated at the position of the detection probes, the genotyping will give a false-negative result. In this study, although HPV 72 and HPV 82 specific probes were included in the kit, negative results were shown. However, the positivity of HPV infection was detected by the universal probe, and the identification of variant forms of HPV 72 and 82 was confirmed by DNA sequencing.

Nowadays, most of the HPV genotyping assays can only detect the most common high-risk and low-risk types but not the unknown-risk types, novel types, or even the intratypic variants. In order to improve the quality of HPV genotyping, next generation sequencing has been developed to sequence cervical DNA. Meiring et al. identified a total of 16 HPV genotypes from 109 cervical specimens from South African HIVpositive women using next generation sequencing, in which four genotypes (HPV-30, 74, 86, and 90) are not included in 
the commercial kit [32]. Nevertheless, this platform is relative expensive, time-consuming, and requires costly equipment. For the GenoFlow test utilized in the present study, the use of PCR with modified PGMY primers targeting the L1 region of HPV followed by hybridization with a universal probe allow rare HPV species or variants outside the genotyping panel to be detected. The Flow-though hybridization system also allows the HPV genotyping to be completed within 3 hours. This rapid test with maximum HPV coverage will be a good alternative in HPV clinical applications.

\section{Conclusions}

In the present study, rare HPV types 30, 91, and 74 and the variant forms of HPV 72 and 82 were successfully detected in Chinese women with low-grade cytological abnormalities by the universal probe available in the GenoFlow HPV Array test. Data on the prevalence of rare HPV types and HPV intratypic variants will assist in clarifying the role of these viruses in cervical intraepithelial lesions and invasive cervical cancer.

\section{Conflict of Interests}

Ngai Na Chloe Co, Lai-On Chu, Joseph K. F. Chow, and Joseph W. O. Tam are the staff of DiagCor Bioscience Inc. Ltd. Enders K. O. Ng is holding an Honorary Assistant Professorship in the University of Hong Kong and is also Senior Scientist at DiagCor Bioscience Inc. Ltd.

\section{References}

[1] E. M. de Villiers, "Cross-roads in the classification of papillomaviruses," Virology, vol. 445, no. 1-2, pp. 2-10, 2013.

[2] J. Doorbar, W. Quint, L. Banks et al., "The biology and life-cycle of human papillomaviruses," Vaccine, vol. 30, supplement 5, pp. F55-F70, 2012.

[3] V. Bouvard, R. Baan, K. Straif et al., "A review of human carcinogens-Part B: biological agents," The Lancet Oncology, vol. 10, no. 4, pp. 321-322, 2009.

[4] J. S. Smith, L. Lindsay, B. Hoots et al., "Human papillomavirus type distribution in invasive cervical cancer and high-grade cervical lesions: a meta-analysis update," International Journal of Cancer, vol. 121, no. 3, pp. 621-632, 2007.

[5] T. Meyer, R. Arndt, E. Christophers et al., "Association of rare human papillomavirus types with genital premalignant and malignant lesions," Journal of Infectious Diseases, vol. 178, no. 1, pp. 252-255, 1998.

[6] S. de Sanjose, W. G. Quint, L. Alemany et al., "Human papilomavirus genotype attribution in invasive cervical cancer: a retrospective cross-sectional worldwide study," The Lancet Oncology, vol. 11, no. 11, pp. 1048-1056, 2010.

[7] A. P. Gurgel, B. S. Chagas, C. do Amaral et al., "Prevalence and genetic variability in capsid L1 gene of rare human papillomaviruses (HPV) found in cervical lesions of women from North-East Brazil," BioMed Research International, vol. 2013, Article ID 546354, 7 pages, 2013.

[8] H. A. Cubie and K. Cuschieri, "Understanding HPV tests and their appropriate applications," Cytopathology, vol. 24, no. 5, pp. 289-308, 2013.
[9] M. Arbyn, G. Ronco, A. Anttila et al., "Evidence regarding human papillomavirus testing in secondary prevention of cervical cancer," Vaccine, vol. 30, supplement 5, pp. F88-F99, 2012.

[10] F. K. Wong, J. C. Ching, and J. K. Chow, "Comparison of the DiagCor GenoFlow human papillomavirus array test and roche linear array HPV genotyping test," The Open Virology Journal, vol. 4, pp. 169-174, 2010.

[11] M. V. Jacobs, P. J. F. Snijders, A. J. C. Van den Brule, T. J. M. Helmerhorst, C. J. L. M. Meijer, and J. M. M. Walboomers, "A general primer GP5+/GP6+-mediated PCR-enzyme immunoassay method for rapid detection of 14 high-risk and 6 low-risk human papillomavirus genotypes in cervical scrapings," Journal of Clinical Microbiology, vol. 35, no. 3, pp. 791-795, 1997.

[12] W. Qu, G. Jiang, Y. Cruz et al., "PCR detection of human papillomavirus: comparison between MY09/MY11 and GP5+/GP6+ primer systems," Journal of Clinical Microbiology, vol. 35, no. 6, pp. 1304-1310, 1997.

[13] H. Lin, Y.-Y. Ma, J.-S. Moh, Y.-C. Ou, S.-Y. Shen, and C.-C. ChangChien, "High prevalence of genital human papillomavirus type 52 and 58 infection in women attending gynecologic practitioners in South Taiwan," Gynecologic Oncology, vol. 101, no. 1, pp. 40-45, 2006.

[14] J. Li, R. Huang, J. E. Schmidt, and Y. L. Qiao, "Epidemiological features of human papillomavirus (HPV) infection among women living in mainland China," Asian Pacific Journal of Cancer Prevention, vol. 14, no. 7, pp. 4015-4023, 2013.

[15] D. Wu, L. Cai, M. Huang, Y. Zheng, and J. Yu, "Prevalence of genital human papillomavirus infection and genotypes among women from Fujian province, PR China," European Journal of Obstetrics Gynecology and Reproductive Biology, vol. 151, no. 1, pp. 86-90, 2010.

[16] S. S. Liu, K. Y. K. Chan, R. C. Y. Leung et al., "Prevalence and risk factors of human papillomavirus (hpv) infection in southern chinese women-a population-based study," PLoS ONE, vol. 6, no. 5, Article ID e19244, 2011.

[17] J. Ye, X. Cheng, X. Chen, F. Ye, W. Lü, and X. Xie, "Prevalence and risk profile of cervical human papillomavirus infection in Zhejiang Province, southeast China: a population-based study," Virology Journal, vol. 7, article 66, 2010.

[18] R. Al-Awadhi, W. Chehadeh, M. Jaragh, A. Al-Shaheen, P. Sharma, and K. Kapila, "Distribution of human papillomavirus among women with abnormal cervical cytology in Kuwait," Diagnostic Cytopathology, vol. 41, no. 2, pp. 107-114, 2013.

[19] Y.-D. Choi, C.-W. Han, W.-J. Chung et al., "Analysis of HPVother samples by performing HPV DNA sequencing," Korean Journal of Pathology, vol. 43, no. 3, pp. 250-253, 2009.

[20] M. L. Tornesello, M. L. Duraturo, P. Giorgi-Rossi et al., "Human papillomavirus (HPV) genotypes and HPV16 variants in human immunodeficiency virus-positive Italian women," Journal of General Virology, vol. 89, no. 6, pp. 1380-1389, 2008.

[21] A. R. Garbuglia, P. Piselli, D. Lapa et al., "Frequency and multiplicity of human papillomavirus infection in HIV-1 positive women in Italy," Journal of Clinical Virology, vol. 54, no. 2, pp. 141-146, 2012.

[22] M. Rahman, T. Sasagawa, R. Yamada, A. Kingoro, H. Ichimura, and S. Makinoda, "High prevalence of intermediate-risk human papillomavirus infection in uterine cervices of kenyan women infected with human immunodeficiency virus," Journal of Medical Virology, vol. 83, no. 11, pp. 1988-1996, 2011. 
[23] M. Longuet, P. Cassonnet, and G. Orth, "A novel genital human papillomavirus (HPV), HPV type 74, found in immunosuppressed patients," Journal of Clinical Microbiology, vol. 34, no. 7, pp. 1859-1862, 1996.

[24] G. M. Andall-Brereton, F. Hosein, R. A. Salas et al., "Human papillomavirus genotypes and their prevalence in a cohort of women in Trinidad," Revista Panamericana de Salud Publica, vol. 29, no. 4, pp. 220-226, 2011.

[25] S. Pande, N. Jain, B. K. Prusty et al., "Human papillomavirus type 16 variant analysis of E6, E7, and L1 genes and long control region in biopsy samples from cervical cancer patients in North India," Journal of Clinical Microbiology, vol. 46, no. 3, pp. 10601066, 2008.

[26] M. R. Pillai, R. Hariharan, J. M. Babu et al., "Molecular variants of HPV-16 associated with cervical cancer in Indian population," International Journal of Cancer, vol. 125, no. 1, pp. 91103, 2009.

[27] V. Cento, M. Ciccozzi, L. Ronga, C. F. Perno, and M. Ciotti, "Genetic diversity of human papillomavirus type 16 E6, E7, and L1 genes in Italian women with different grades of cervical lesions," Journal of Medical Virology, vol. 81, no. 9, pp. 1627-1634, 2009.

[28] Z. Sun, J. Liu, G. Wang, W. Zhou, C. Liu, and Q. Ruan, "Variant lineages of human papillomavirus type 18 in Northeast China populations characterized by sequence analysis of E6, E7, and L1 regions," IInternational Journal of Gynecological Cancer, vol. 22, no. 6, pp. 930-936, 2012.

[29] B. J. Kocjan, K. Seme, M. Cimerman, A. Kovanda, M. Potočnik, and M. Poljak, "Genomic diversity of human papillomavirus (HPV) Genotype 38," Journal of Medical Virology, vol. 81, no. 2, pp. 288-295, 2009.

[30] Z. Sun, Z. Lu, J. Liu et al., "Genomic polymorphism of human papillomavirus type 52 in women from Northeast China," International Journal of Molecular Sciences, vol. 13, no. 11, pp. 1496214972, 2012.

[31] L. H. S. Oliveira, L. S. Santos, E. F. Augusto, and F. P. G. Neves, "Identification of human papillomavirus type $53 \mathrm{L1}$, E6 and E7 variants in isolates from Brazilian women," Infection, Genetics and Evolution, vol. 12, no. 1, pp. 71-76, 2012.

[32] T. L. Meiring, A. T. Salimo, B. Coetzee et al., "Next-generation sequencing of cervical DNA detects human papillomavirus types not detected by commercial kits," Virology Journal, vol. 9, p. 164, 2012. 

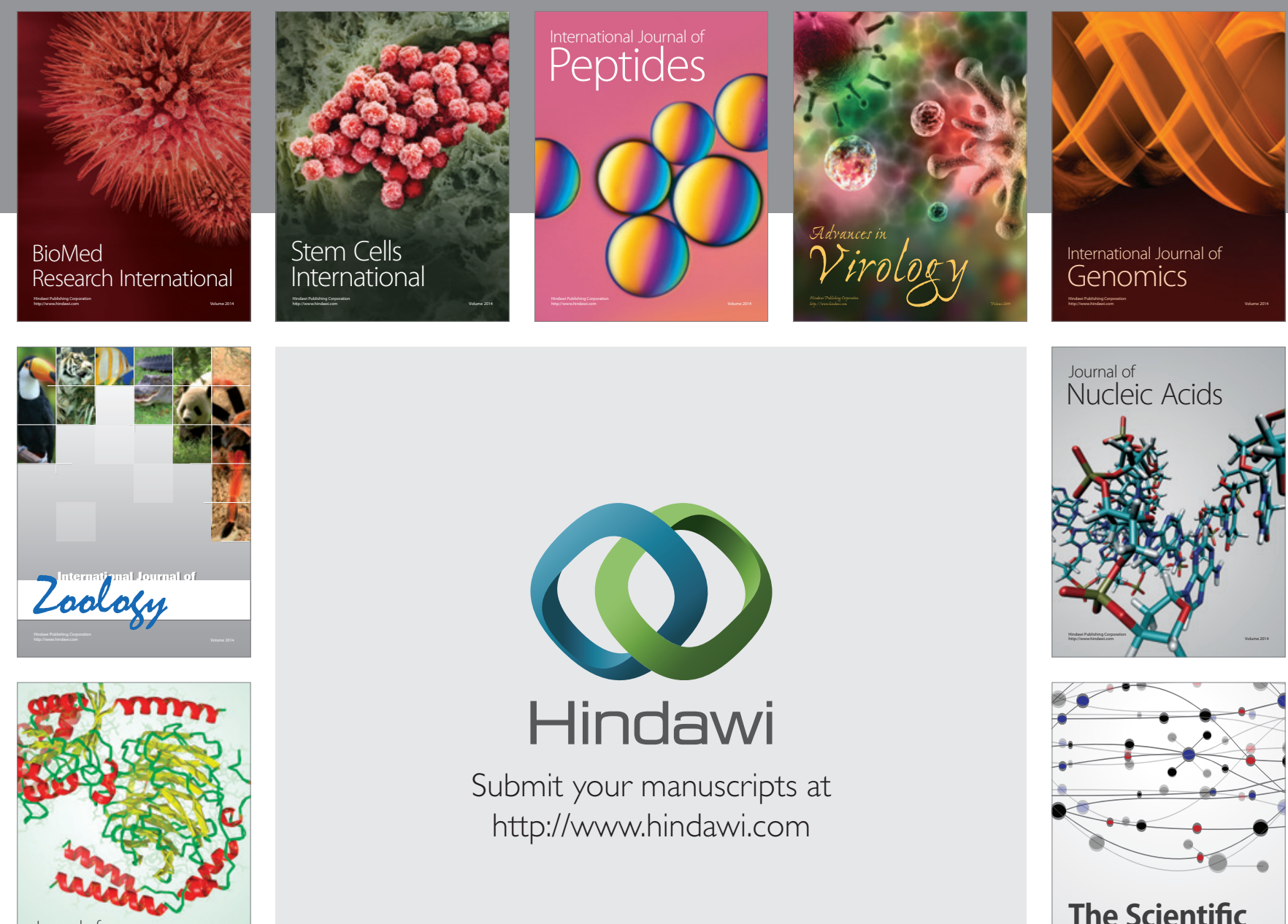

Submit your manuscripts at

http://www.hindawi.com

Journal of
Signal Transduction
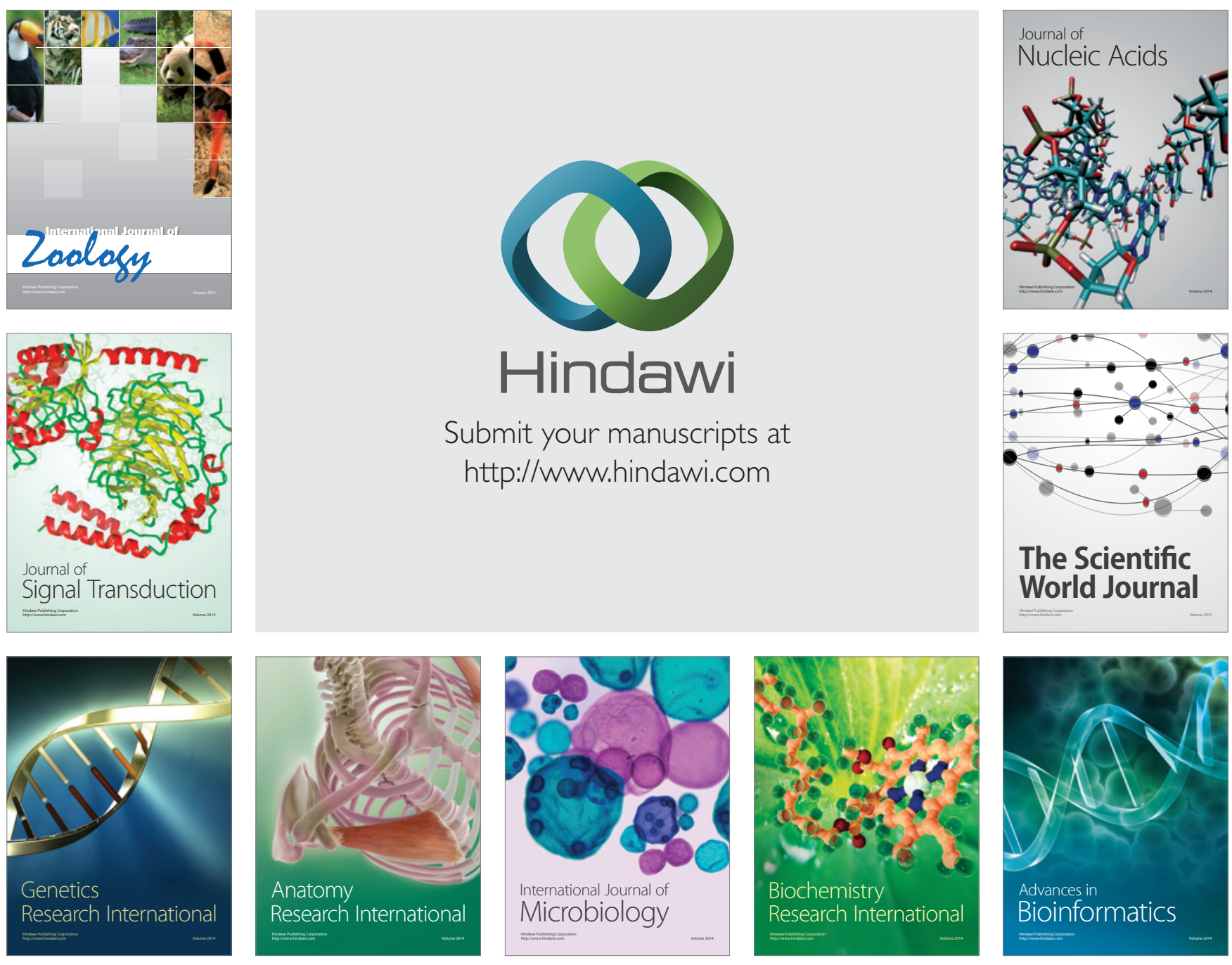

The Scientific World Journal
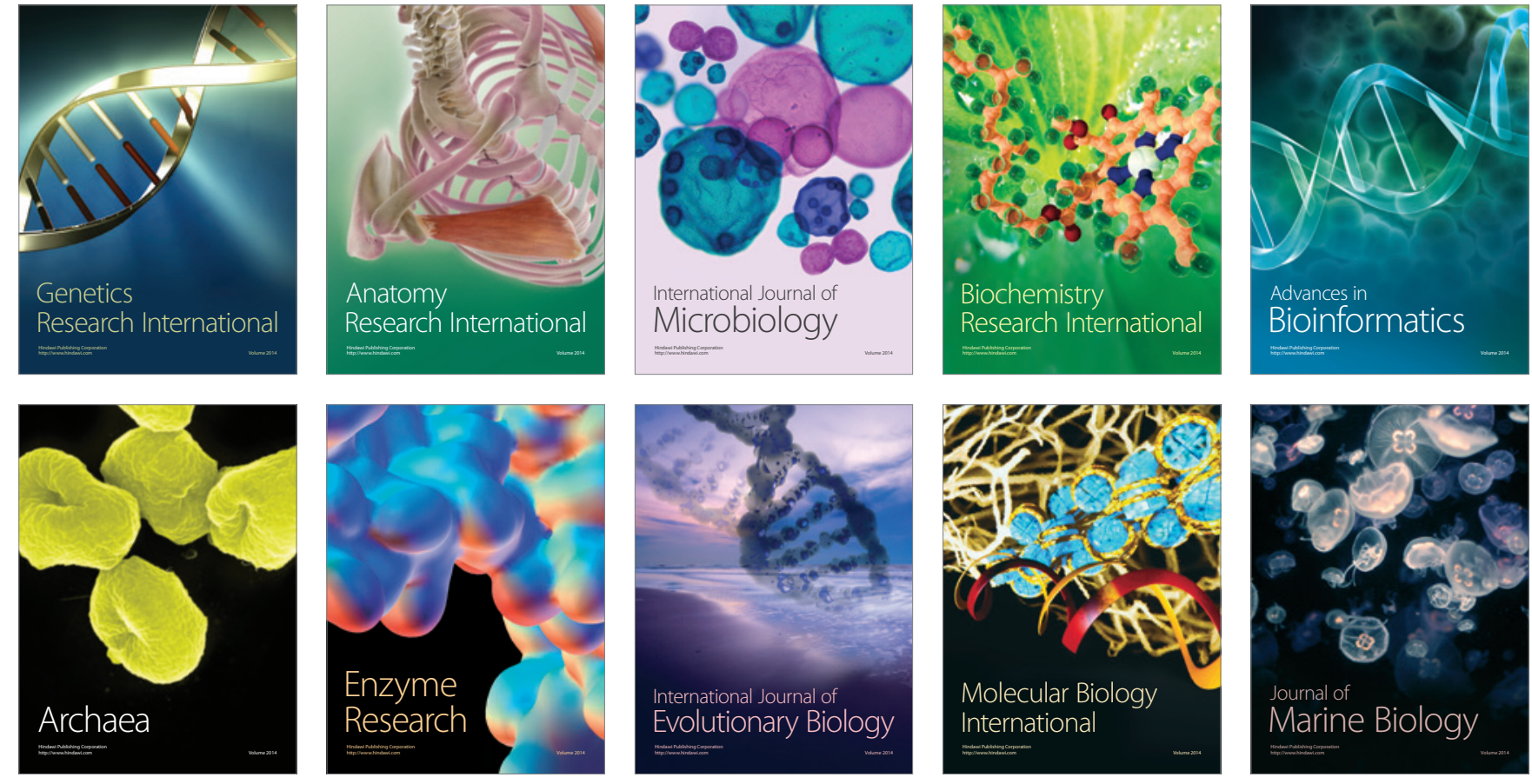